\title{
Effects of an outpatient service holistic rehabilitation program in a case of pulmonary atresia
}

\author{
Maurizio Bussotti ${ }^{1}$, Marinella Sommaruga ${ }^{2}$, Patrycja Krasinska ${ }^{1}$, Laura Adelaide Dalla Vecchia ${ }^{1}$ \\ ${ }^{1}$ Cardiorespiratory Rehabilitation Department, IRCCS Maugeri Clinical Scientific Institutes, Milan; ${ }^{2}$ Psychology Unit, \\ IRCCS Maugeri Clinical Scientific Institutes, Milan, Italy
}

In memory of Sara

\begin{abstract}
A 42-year-old woman affected by pulmonary atresia came to our attention complaining of dyspnea and fatigue for minimal efforts with important desaturation.

After assessing her basal functional capacity with a cardiopulmonary exercise test, the patient was enrolled in an extremely individualized rehabilitation program, which entailed a discrete improvement in the quality of life indices, in the absence of side effects.
\end{abstract}

\author{
Correspondence: Maurizio Bussotti, Cardiorespiratory Rehabilitation \\ Department, IRCCS Maugeri Clinical Scientific Institutes, Via Camaldoli \\ 64, 20138 Milan, Italy. \\ Tel. +39.02.50725245. \\ E-mail: maurizio.bussotti@icsmaugeri.it
}

Key words: Pulmonary atresia; cardiorespiratory rehabilitation; cardiopulmonary exercise test.

Authors' contributions: MB, MS, PK, contribution to the interpretation of the case, and to the conception and design of this paper; LADV, contribution to the drafting the article, manuscript critical revision. All the Authors gave their final approval of the version to be published, and the agreement to be accountable for all aspects of the work.

Conflict of interest: The authors declare that they have no competing interests, and all authors confirm accuracy.

Ethics approval and consent to participate: No ethical committee approval was required for this case report by the Department, because this article does not contain any studies with human participants or animals.

Availability of data and materials: All data underlying the findings are fully available.

Received for publication: 11 September 2020.

Accepted for publication: 14 December 2020.

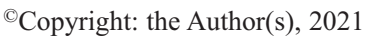

Licensee PAGEPress, Italy

Monaldi Archives for Chest Disease 2021; 91:1605

doi: 10.4081/monaldi.2021.1605

This article is distributed under the terms of the Creative Commons Attribution Noncommercial License (by-nc 4.0) which permits any noncommercial use, distribution, and reproduction in any medium, provided the original author(s) and source are credited.
This paper shows that even patients with extremely severe forms of congenital heart disease, when clinical stable, can undergone a tailored cardiorespiratory rehabilitation program. This must be carried out in a monitored environment and under the supervision of expert personnel.

\section{Case Report}

A 42-year-old woman presented with the following medical history: at the age of 13 months, during a flu syndrome, she developed exertional cyanosis. A tetralogy of Fallot was suspected. Three months later, she underwent a cardiac catheterization that showed pulmonary atresia (PA), an interventricular defect (IVD), a right-sided aortic arch, the presence of some non-confluent pulmonary branches, and a pulmonary flow supported by two collaterals originating from the thoracic aorta at T5 level (the first collateral directed to the left and upwards had a severe stenosis shortly after the origin and supplied the two upper lobes, the second one was greatly dilated and supplied the lower left lobe). At the age of 2 years, she underwent a first palliative intervention that produced a central shunt interposing a duct between the ascending aorta and the major collateral connected to the left lung.

When the patient was 7 years old, because of a central occlusion of the shunting duct, she underwent a modified right BlalockTaussig palliative intervention (connection of the right subclavian artery to the pulmonary arteries through a synthetic tube). Despite multiple hospitalizations for lung infections, this girl grew up and decided to become a doctor. She graduated in 1994 and then specialized in Cardiology at the University of Milan.

The last thoracic MRI, at 41 years of age, confirmed a right ventricular outflow atresia with the absence of both the pulmonary arterial trunk and any significant pulmonary branches on the right, some non-confluent pulmonary branches on the left with two large, but stenotic systemic pulmonary collaterals on the left originating from the descending aorta, a large systemic pulmonary collateral circulation on the right with a sub-diaphragmatic origin and a well-developed branch coming from the right subclavian artery, an important dilatation of the aortic bulb and the ascending aorta with a right-sided aortic arch, a moderate regurgitation of a tricuspid aortic valve, a moderately dilated and hypertrophic right ventricle with a preserved systolic function, a wide IVD from septal misalignment with an overriding aorta causing a significant left-to-right shunt (Figure 1).

The combination of PA, IVD and major aortopulmonary collateral arteries is the most severe form of tetralogy of Fallot. During the intrauterine development, the absence of both an anterograde flow from the right ventricle and a flow through the arterial duct 
leads to the development of different types of aortopulmonary collaterals. These differently supply the lung segments, with a potentially infinite variety of pulmonary vascularization states at birth. Most collateral arteries usually originate from the descending thoracic aorta; however they can also come from the aortic arch, the subclavian artery, the distal thoracic aorta, the internal mammary artery, and the left coronary artery. Identifying the sources of pulmonary blood flow and creating appropriate pulmonary arborization are paramount to the management of this anomaly [1].

Throughout their life, children with PA develop severe cyanosis, symptoms related to heart failure and failure to thrive. The natural history of this heart disease carries high mortality with a $60 \%$ survival at 1 year, a survival of less than $50 \%$ at 10 years, and less than $20 \%$ at 20 years [2].

We first evaluated this patient when she self-referred to our rehabilitation cardiology center after gaining information from PubMed [3]. Physical examination was notable for important digital and mucous membranes cyanosis, arrhythmic cardiac sounds with frequent premature beats, a 3/6 systolic murmur at the apex, mild lower limb edema, and hepatomegaly. EKG showed sinus rhythm, pulmonary $\mathrm{P}$ waves and right bundle branch block. She was taking furosemide, spironolactone and warfarin.

Despite many years of experience in the rehabilitative treatment of severe forms of pre-capillary pulmonary hypertension, the patient's request represented a novelty. Therefore, a complete basal evaluation of her clinical condition and functional capacity was first performed. An echocardiographic examination showed a dilated aortic root $(\mathrm{mm})$, ascending tract $(50 \mathrm{~mm})$ and arch $(34$ $\mathrm{mm}$ ), a moderate aortic regurgitation into the right ventricle (RV), a right atrial enlargement $(\mathrm{ml})$, the presence of a large perimembranous IVD with a bidirectional shunt; normal left ventricular (LV) volumes and wall thickness, but diffuse hypokinesia determining a moderately reduced LV ejection fraction of $43 \%$, a dilat-

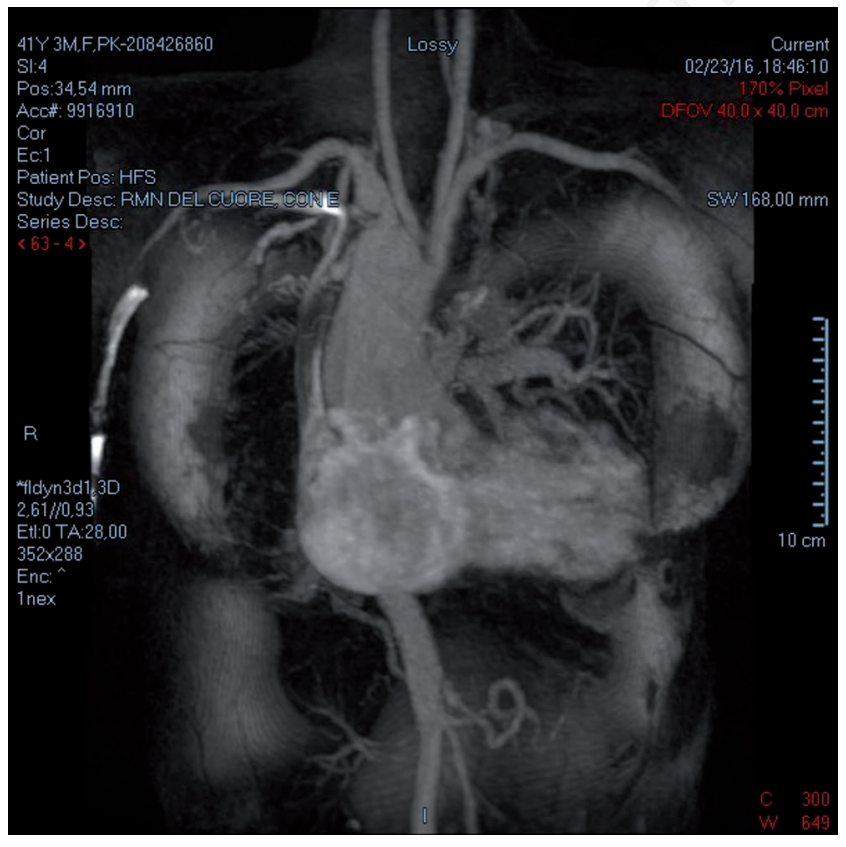

Figure 1. The last thoracic magnetic resonance imaging performed by the patient confirming a right ventricular outflow atresia with the absence of both the pulmonary arterial trunk and any significant pulmonary branches on the right. ed and moderate hypertrophic RV with moderately reduced systolic function (TAPSE mm), a moderate tricuspid regurgitation, the absence of the RV outflow tract and pulmonary valve apparatus, a dilated and non-collapsible inferior vena cava, normal mitral valve apparatus and normal left atrial volume.

A cardiopulmonary exercise test (CPET), as recommended $[4,5]$, was used to evaluate the functional status. The CPET was conducted on a cycloergometer using a ramp protocol (4 Watt/min), maximal for the respiratory quotient (RQ) reached, it was interrupted because of muscle exhaustion at the load of 28 Watt. During exertion, frequent isolated and monomorphic ventricular premature beats were recorded. An early anaerobic threshold was observed, with an oxygen consumption at the peak exercise (peak $\mathrm{VO}_{2}$ ) equal to $21 \%$ of the predicted value. The slope of the relation between the increase of $\mathrm{VO}_{2}$ and the increase of the work rate $\left(\Delta \mathrm{VO}_{2} / \Delta \mathrm{WR}\right.$ slope $)$ was $5.8 \mathrm{ml} / \mathrm{watt} / \mathrm{min}$ while the pulse $\mathrm{O}_{2}$ at peak (peak $\mathrm{VO}_{2} /$ heart rate) was $5.9 \mathrm{ml} /$ beat. A severely increased ratio between ventilation and carbon dioxide production $\left(\mathrm{VE} / \mathrm{VCO}_{2}=81\right)$ with severe desaturation (descent down to $65 \%$ ) were also measured indicating a major increase in ventilatory inefficiency (Figure 2). Overall, we concluded that there was evidence of a severe limitation of exercise capacity with multiple etiological mechanisms, but a so high relationship $\mathrm{VE} / \mathrm{VCO}_{2}$, with a negative intercept, would confirm the prevalence of a pulmonary vascular limitation. The distance at the 6-minute walking test (6MWT) was $300 \mathrm{~m}$ (45\% of predicted) and the Maximal Inspiratory Pressure (MIP) was $101 \mathrm{cmH}_{2} \mathrm{O}$.

We agreed to enroll the patient in a rehabilitation program (RP), that was individually tailored consisting of muscle strengthening exercises with dumbbell $(0.5 \mathrm{~kg})$, inspiratory muscles training using the Threshold IMT ${ }^{\circledR}$, controlled breathing exercises, submaximal aerobic training with cycloergometer and treadmill and mental gait training in the hospital garden at variable speeds and slopes. All

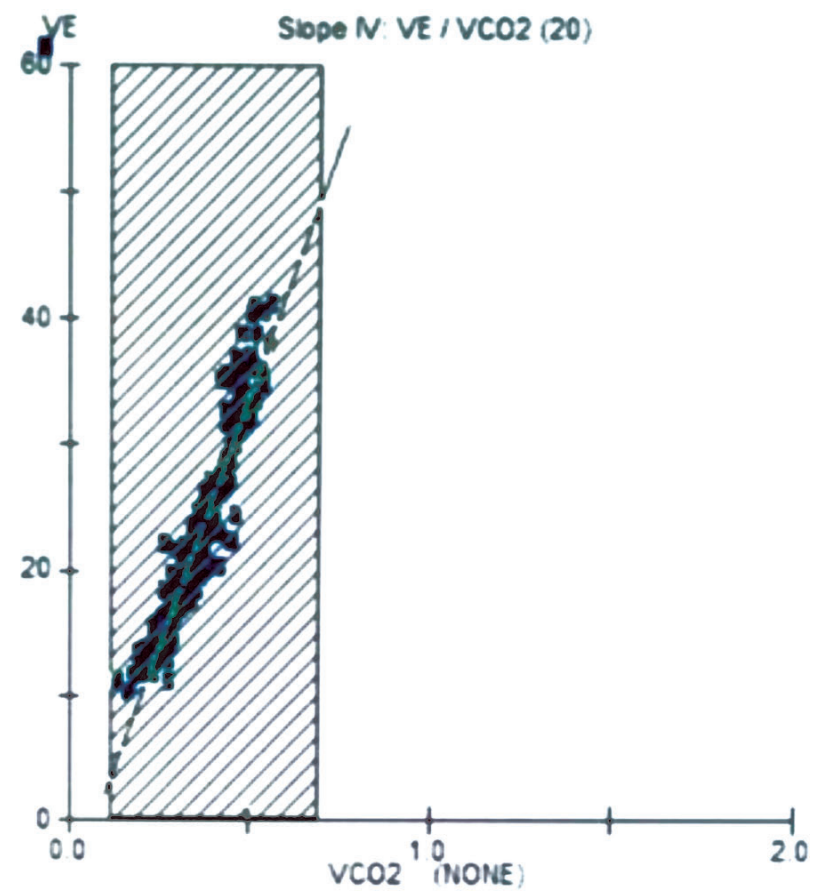

Figure 2. A severely increased ratio between ventilation and carbon dioxide production at the CPET. 
these activities were performed as described before [6], according to a schedule carried out in agreement with the patient, maintaining a low intensity in terms both of the exercise load and of the number of sessions per week. Actually the aerobic training was conducted at a constant workload of 10 Watt for $30 \mathrm{~min}$, established on the basis of anaerobic threshold determined at the preliminary CPET.

Between June and December 2017, the patient completed 30 sessions at our outpatient clinic. The RP was interrupted for a month in August (patient's holidays) and for 20 days in October due to an acute bronchitis. During the training no serious adverse events (syncope, severe arrhythmias, emophyte) were observed. At the end of the RP, the 6MWT and the MIP were repeated, with a walked final distance of $283 \mathrm{~m}$ (with a maximal nadir of $\mathrm{SpO}_{2}$ of $69 \%$ ), and a measure of $114 \mathrm{cmH}_{2} \mathrm{O}$ for MIP at the discharge.

At each session, the patient was supported by a clinical psychologist. The initial clinical psychometric and psychological evaluation showed slight anxiety (as quantified by the Hospital Anxiety and Depression Scale (HADS) [7]: anxiety 8/21; depression $7 / 21$ ), but a high perceived distress emerged, associated with the history of the disease. Appropriate disease awareness, adequate internal resources and good family support were also noted. The initial psychometric assessment of Quality of Life (EuroQol-5D) [8] showed a self-perception of a moderate health's state $(55 / 100$ at the Visual Analog Scale). The psychological intervention had the aim: i) to assess and to manage emotional issues (anxiety, depression, and panic attacks especially); ii) to enhance knowledge, awareness, and acceptance of the illness; and iii) to allow personal, interpersonal, family, social, and occupational adaptation to the disease [9]. The pre-discharge psychometric evaluation indicated a slight worsening of the anxious symptoms, in the presence, however, of a better awareness and new disease management skills (HADS: anxiety 10/21; depression 5/21). The EuroQol-5D at discharge indicated the perception of an improvement in her health's condition (Visual Analogical Scale 75/100).

In the following months, the patient resumed exercising as an outpatient cardiologist, keeping herself in stable health. Although she no longer participated in a rehabilitation program at our Center, she maintained stable contact with our team, periodically visiting us or meeting via chat with our physiotherapists to periodically update her rehabilitation program.

In January 2019, we learned that the patient had been hospitalized and died of pneumonia.

\section{Discussion}

In this case report, we present a holistic rehabilitation approach to a highly disabling heart disease that led to a significant improvement of quality of life without any side effect.

PA is an extremely complex congenital heart disease burdened by a high mortality within the first 20 years of life. These patients experience from the first years of life a great limitation of their ability to carry out the common daily activities, in face of important dyspnea and cyanosis. A constant fatigue, numerous physical limitations, frequent respiratory complications and hospital admissions, despite the complex cardiac surgery they go through as children, represent a heavy burden for these patients [1]. In addition, the awareness of a poor prognosis inevitably leads to extreme psychological fragility [2].

Consensus reports have stated that exercise should be encouraged and regularly performed in these patients, but this is not common practice [10]. A rehabilitation program, that includes nutri- tional and psychological support, aerobic symptom-limited training, muscle strengthening exercises, inspiratory muscles training and controlled breathing exercises, can be beneficial in these patients, as already suggested/demonstrated in patients with advanced forms of cardiorespiratory insufficiency who improve their functional capacity and quality of life [10]. It should be emphasized that such RP should be performed under strict monitoring and by experienced personnel $[3,6,11]$.

\section{Conclusions}

Rehabilitation programs in patients with congenital heart disease should always be encouraged, even in the most complex forms and in the most severe clinical pictures. In agreement with the patient, each program should be tailored and achieved through a multidisciplinary approach.

From this approach, we can expect an improvement at least in the quality of life indices, if not necessarily in the exercise performance.

\section{References}

1. Presnell LB, Blankenship A, Cheatham SL, et al. An overview of pulmonary atresia and major aortopulmonary collateral arteries. World J for Ped and Cong Heart Surg 2015;6:630-9.

2. Leonard H, Derrick G, O'Sullivan J, Wren C. Natural and unnatural history of pulmonary atresia. Heart 2000;84:499503.

3. Dalla Vecchia LA, Bussotti M. Exercise training in pulmonary arterial hypertension. J Thorac Dis 2018;10:508-21.

4. Miliaresis C, Beker S, Gewitz M. Cardiopulmonary stress testing in children and adults with congenital heart disease. Cardiol Rev 2014;22:275-8.

5. Inuzuka R, Diller GP, Borgia F, et al. Comprehensive use of cardiopulmonary exercise testing identifies adults with congenital heart disease at increased mortality risk in the medium term. Circulation 2012;125:250-9.

6. Bussotti M, Gremigni P, Pedretti RFE, et al. Effects of an outpatient service rehabilitation programme in patients affected by pulmonary arterial hypertension: An observational study. Cardiovasc Hematol Disord Drug Targets 2017;17:3-10.

7. Zigmond AS, Snaith RP. The hospital anxiety and depression scale. Acta Psychiatr Scand 1983;67:361-70.

8. Balestroni G, Bertolotti G. [EuroQol-5D (EQ-5D): an instrument for measuring quality of life].[Article in Italian]. Monaldi Arch Chest Dis 2012;78:155-9.

9. Bussotti M, Sommaruga M. Anxiety and depression in patients with pulmonary hypertension: impact and management challenges. Vasc Health Risk Manag 2018;14:349-60.

10. Duppen N, Takken T, Hopman MT, et al. Systematic review of the effects of physical exercise training programmes in children and young adults with congenital heart disease. Int J Cardiol 2013;168:1779-87.

11. Duppen N, Etnel JR, Spaans L, et al. Does exercise training improve cardiopulmonary fitness and daily physical activity in children and young adults with corrected tetralogy of Fallot or Fontan circulation? A randomized controlled trial. Am Heart J 2015;170:606-14. 